OPEN ACCESS

Edited by:

Masahiro Ohsawa,

Nagoya City University, Japan

Reviewed by:

Yoshinobu Nakada,

Tokai University School of

Medicine, Japan

Nobuaki Michihata,

The University of Tokyo, Japan

*Correspondence:

Kasumi Satoh

satohkasumi19900114@gmail.com

Specialty section:

This article was submitted to

Clinical Nutrition,

a section of the journal

Frontiers in Nutrition

Received: 29 July 2021 Accepted: 13 September 2021

Published: 11 October 2021

Citation:

Satoh K and Nakae H (2021) Daijokito Administration in Critically III Patients Increasing the Stool Volume: A Retrospective Observational Study.

Front. Nutr. 8:749570.

doi: 10.3389/fnut.2021.749570

\section{Daijokito Administration in Critically III Patients Increasing the Stool Volume: A Retrospective Observational Study}

\author{
Kasumi Satoh* and Hajime Nakae \\ Advanced Emergency and Critical Care Center, Akita University Hospital, Akita, Japan
}

Introduction: Daijokito, a traditional Japanese herbal medicine (Kampo), has been used to treat abdominal distention of the middle yang stage pattern. The use of Daijokito has not been thoroughly investigated in critical care. To investigate a new Kampo approach to defecation control in critically ill patients, our study aimed to assess the effects of Daijokito on fecal management.

Methods: We analyzed 30 consecutive patients treated with Daijokito in the intensive care unit (ICU) between March 2017 and February 2021. The eligibility criteria were patients who were newly prescribed Daijokito in the ICU during the study period. Exclusion criteria were patients who were started on other laxatives within one day of beginning Daijokito. The study's primary outcome was defecation volume three days before and three days after starting Daijokito. We recorded the most dominant stool quality within three days after the start of Daijokito.

Results: Twenty-one patients were included in the analysis. The median age was 69.0 years, and the median sequential organ failure assessment score on admission to the ICU was 6.0. Major diseases included trauma, pancreatitis, and burns. Administration of Daijokito resulted in defecation in 17 of twenty-one patients (81.0\%). Comparison of defecation volume between 3 days before Daijokito administration and three days, including the day of Daijokito administration, showed that defecation volume increased significantly after Daijokito administration, with a median of 0 to $360 \mathrm{~g}(p<0.001)$. At the three-day follow-up, six of 17 (35.3\%) patients defecated on the day of Daijokito administration, and nine (52.9\%) defecated on the day after administration. One patient was judged to have excessive defecation, and Daijokito administration was discontinued. Stool quality was normal in one (5.9\%) of the 17 patients, soft-formed in two (11.8\%), loose-unformed in 11 (64.7\%), and liquid in three (17.6\%).

Discussion: Daijokito administration in critically ill patients caused defecation in $81 \%$ of the patients and significantly increased stool volume. The novelty of this study is that it sheds light on the Kampo treatment of defecation control in critically ill patients. In addition to the present report, further studies are warranted to quantify the therapeutic efficacy and safety of Daijokito.

Keywords: Kampo, critical care, intensive care units, constipation, laxatives 


\section{INTRODUCTION}

Most doctors in Japan are reported to use Kampo, traditional Japanese herbal medicine (1). The Kampo medicine Daijokito (DJT) is composed of the following herbs: Magnolia bark, immature orange, rhubarb rhizome, and anhydrous mirabilitum (Figure 1). In Kampo medicine, magnolia bark and immature orange regulate and normalize the flow of qi. Rhubarb rhizome and anhydrous mirabilitum have purgative properties and remove heat toxins [Traditionalmedicinemodule1:TM1] in the intestinal tract. Therefore, DJT has been used to treat abdominal distention, constipation, wheezing, and psychological symptoms in the middle yang stage pattern. Chapter 208 in Shanghanlun says that "When in middle yang stage pattern, the pulse is slow, thought there is sweating, but aversion to cold is absent, there will be generalized heaviness, shortness of breath, abdominal fullness, panting, and tidal heat effusion, which means the exterior ${ }^{\text {[TM1] }}$ is about to resolve and one can attack the interior ${ }^{[\mathrm{TM} 1]}$. Sweat streaming from the extremities indicates that the stool is already hard, the DJT governs." DJT is used in clinical settings to treat significant constipation, hypertension, neurosis, and food poisoning. It has also been reported as a treatment for acute pancreatitis, paralytic ileus, and tetanus in intensive care medicine (2-4).

Among Kampo medicines for gastrointestinal motility, Daikenchuto is the most investigated. Some randomized controlled trials have shown that Daikenchuto significantly enhanced ascending colon emptying compared to a placebo (5); Daikenchuto reduced postoperative ileus surgery and postoperative ileus recurrence (6). In the Japanese guidelines for nutrition support therapy in critically ill patients, Daikenchuto is expected to be a potentially effective treatment for improving gastrointestinal motility (7). However, DJT has not been well investigated. DJT contains rhubarba with stimulant laxative action (8); thus, compared with Daikenchuto, which does not include rhubarba, DJT should be a more potent agent to induce intestinal motility.

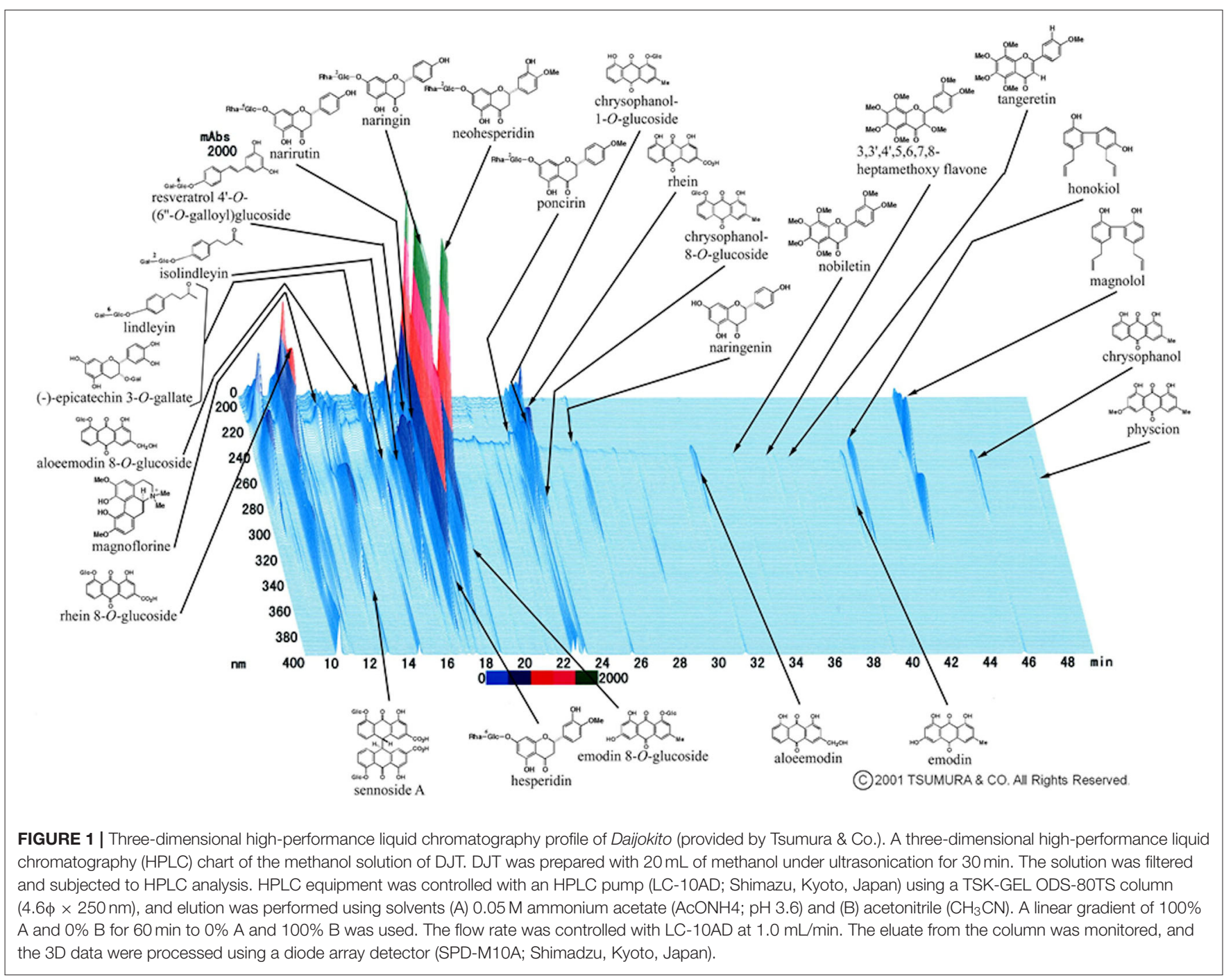




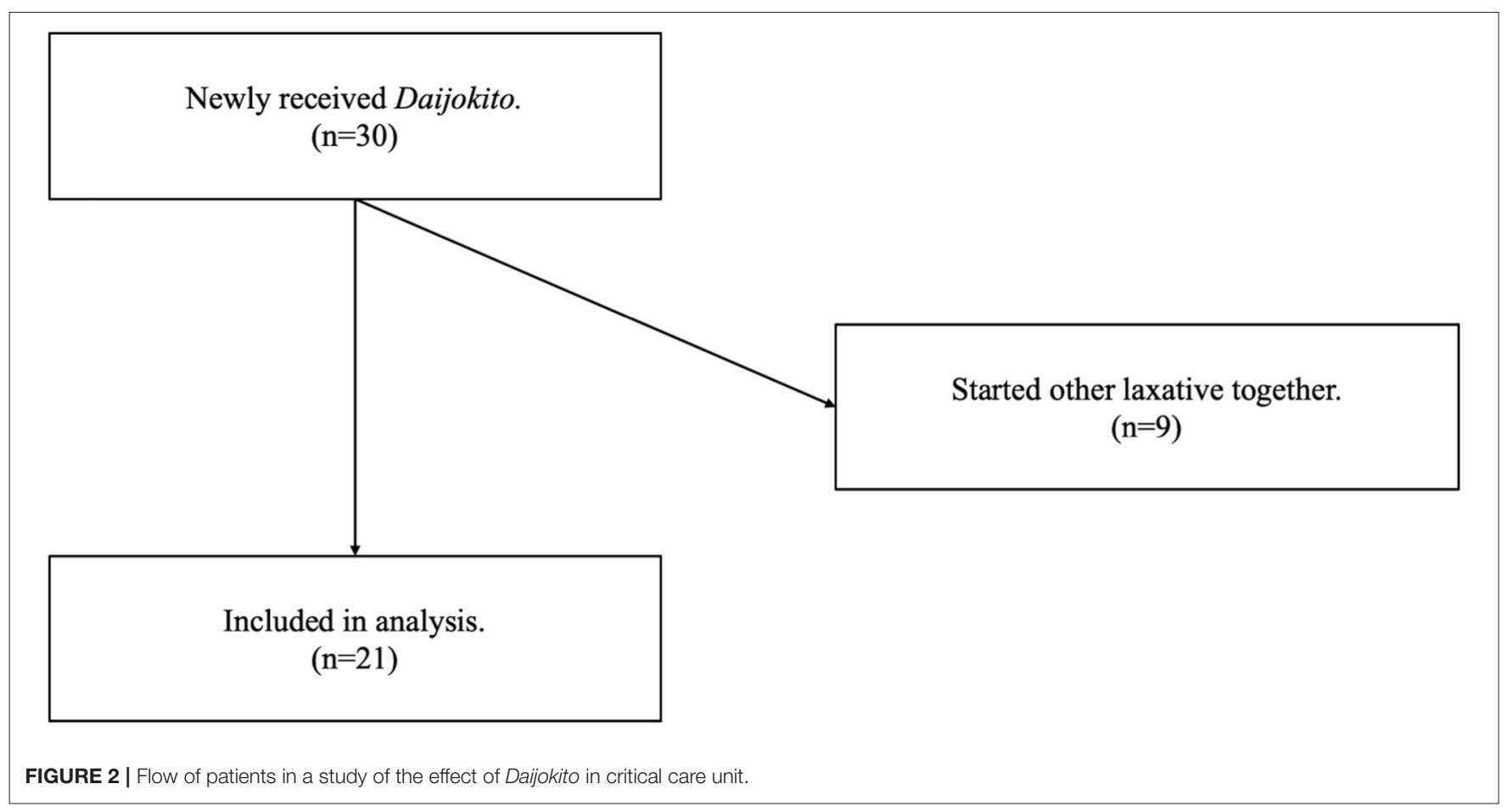

Constipation is frequently reported in $50-83 \%$ of critically ill patients $(9,10)$. Patients with more severe illness are more likely to have constipation (11). Constipation is reported to be associated with adverse events such as ventilator weaning failure (9) and delirium (12). Thus, defecation control in critically ill patients has important implications.

To investigate a new Kampo approach to defecation control in critically ill patients, our study aimed to assess the effect of DJT on fecal management in critically ill patients.

\section{MATERIALS AND METHODS Study Design and Participants}

We analyzed 30 consecutive patients treated with DJT in the intensive care unit (ICU) of Akita University Hospital between March 2017 and February 2021. Akita University Hospital is a tertiary care hospital in a rural area and has 16 ICU beds. This study was a retrospective observational cohort study and conformed to the principles of the Declaration of Helsinki. The ethics committee of Akita University Hospital approved the study protocol. The need for informed consent was admitted waiving because of the observational nature of the study and the requirement for no treatments beyond the daily clinical practice. The eligibility criteria were patients who were newly prescribed DJT in the ICU during the study period. The exclusion criteria were patients who were started on other laxatives within one day of beginning DJT.

\section{DJT}

The decision of who to administer DJT was left to the clinician. Clinicians often initiate DJT based on the following factors: small volume of defecation, prolonged absence of stool, abdominal physiological findings, and abdominal radiographic findings. DJT was administered three times daily, one pack at a time via nasogastric tube. The DJT used in this study was produced by Tsumura \& Co. (Tokyo, Japan) (8).

\section{Outcome}

The study's primary outcome was defecation volume three days before and three days after starting DJT. In addition, the number of constipated days, number of days between the start of DJT and defecation, stool quality, and survival at discharge were recorded. If a new laxative was added before defecation, we judged that DJT was ineffective and recorded the stool volume as zero. Stool quality was classified into four categories: normal, soft-formed, loose-unformed, and liquid. We recorded the most dominant stool quality within three days after the start of DJT.

\section{Statistical Methods}

To determine if there was a difference in each variable between the pre - and post-DJT administration, we used the Wilcoxon signed-rank test to assess the significance level at 5\%. There were no missing data. Statistical analyses were conducted using Stata $\mathbb{R}$ software (version 16.1; StataCorp, College Station, Texas, USA). Significance was defined as a two-sided $p$-value of $<0.05$.

\section{RESULTS}

Of the 30 patients newly received DJT in the ICU, nine were met the exclusion criteria because they started other laxative treatments at the same time. Therefore, we analyzed twentyone patients (Figure 2). The median age was 69.0 years, and the proportion of male patients was $52.4 \%$. The median SOFA score (13) on admission to the ICU was 6.0, and the median 
TABLE 1 | Characteristics of the patients included in the current study.

\begin{tabular}{|c|c|c|}
\hline & Variables & \\
\hline Age (years old) & $69(57-80)$ & \\
\hline Male & $11 / 21(52.4 \%)$ & \\
\hline SOFA score & $6(4-8)$ & \\
\hline Constipation day (days) & $2(1-3)$ & \\
\hline \multirow[t]{11}{*}{ Diagnosis } & Trauma & $4(19.0 \%)$ \\
\hline & Pancreatitis & $3(14.3 \%)$ \\
\hline & Burn & $3(14.3 \%)$ \\
\hline & Sepsis & $2(9.5 \%)$ \\
\hline & Tetanus & $2(9.5 \%)$ \\
\hline & Intoxication & $2(9.5 \%)$ \\
\hline & Heatstroke & $1(4.8 \%)$ \\
\hline & Carbon dioxide narcosis & $1(4.8 \%)$ \\
\hline & Acute heart failure & $1(4.8 \%)$ \\
\hline & Neuroleptic Malignant Syndrome & $1(4.8 \%)$ \\
\hline & Post-cardiac arrest syndrome & $1(4.8 \%)$ \\
\hline Opioid use & 18/21 (85.7\%) & \\
\hline Enteral nutrition use & 20/21 (95.2\%) & \\
\hline
\end{tabular}

Data are expressed as medians (interquartile ranges) for continuous variables and numbers (\%) for categorical variables. SOFA, Sequential Organ Failure Assessment.

duration of no defecation before DJT administration was two days. The patients' major diseases included trauma, pancreatitis, burn, sepsis, tetanus, and intoxication. The patient characteristics are shown in Table 1. No patients underwent abdominal surgery.

Of the twenty-one patients, we attempted to control defecation in 13 patients $(61.9 \%)$ with other laxatives before DJT, five patients $(23.8 \%)$ received one laxative, five patients $(23.8 \%)$ received two laxatives, two patients $(9.5 \%)$ received three laxatives, and one patient (4.8\%) received four laxatives. As for the type of laxative, oral sodium picosulfate solution was used for six (28.6\%), oral Daikenchuto, a herbal medicine, for six (28.6\%), bisacodyl suppository for five (23.8\%), oral naldemedine tosilate for three (14.3\%), oral sennoside for two (9.5\%), oral magnesium citrate solution, oral magnesium oxide, and glycerin enema for one $(4.8 \%)$. As these drugs were considered inadequate, DJT was administered.

Administration of DJT resulted in defecation in 17 of 21 patients (81.0\%). Comparison of defecation volume between 3 days before DJT administration (Pre-DJT group) and three days, including the day of DJT administration (Post-DJT group) showed that defecation volume increased significantly after DJT administration with a median of $0 \mathrm{~g}$ (interquartile range [IQR], $0-100)$ in the Pre-DJT group and $360 \mathrm{~g}$ (IQR, 148-560) in the Post-DJT group ( $p<0.001$, Figure 3$)$.

At the three-day follow-up, including the day of DJT administration, six of $17(35.3 \%)$ patients defecated on the day of DJT administration, nine $(52.9 \%)$ defecated on the day after administration, and two (11.8\%) defecated two days after administration. One patient was judged to have excessive defecation, and DJT administration was discontinued on the second day. Stool quality was normal in one $(5.9 \%)$ of the 17 patients, soft-formed in two (11.8\%), loose-unformed in 11 (64.7\%), and liquid in three (17.6\%). There were four deaths out of twenty-one patients at discharge, with a mortality rate of $19.1 \%$.

\section{DISCUSSION}

DJT administration in critically ill patients causes defecation in $81 \%$ of patients and significantly increases stool volume. Therefore, DJT is expected to improve lower gastrointestinal motility in intensive care units. Most of the defecation occurred on the day of administration or the next day, and loose-unformed was the most common stool quality. This study is novel as it is one of the first to shed light on the Kampo treatment of defecation control in critically ill patients. To the best of our knowledge, this is the first study to focus on DJT.

First, at the 3-day follow-up after DJT administration, 81\% of patients showed an increase in stool volume, and more than half of these patients had bowel movement the day after DJT administration. The median stool volume increased from $0 \mathrm{~g}$ per 3 days to 360 g every 3 days. Few previous studies have evaluated the effect of individual laxatives on defecation volume in critically ill patients. For instance, in patients with constipation with multiple organ failure, lactulose promoted defecation in $69 \%$ of patients (median time to defecation was $36 \mathrm{~h}$ ), and polyethylene glycol stimulated defecation in $74 \%$ of patients (median time to defecation was $44 \mathrm{~h}$ ) (14). DJT may be competitive with these typical laxatives. Moreover, about $60 \%$ of our patients were administered DJT because other laxatives were ineffective. We propose DJT as a treatment option for refractory lower gastrointestinal motility failure in critically ill patients.

Second, loosely unformed stool was the most common stool quality, and about $82.4 \%$ of the patients presented with diarrheal stools. Almost all of our patients received parenteral nutrition. Considering that diarrhea occurred in $18 \%$ of patients receiving enteral feeding (15), the incidence of diarrhea in patients receiving DJT was high. However, only one patient $(5.9 \%)$ discontinued DJT administration because of clinically determined excessive defecation, and DJT was unlikely to cause diarrhea with adverse effects, such as water and electrolyte imbalance.

The basic strategy for treating constipation is stool softening by regulating the intestinal tract's water content and enhancing bowel motility by stimulating the intestinal mucosa. The anhydrous mirabilitum in DJT has a stool softening effect, and rhubarb has a hypermotility effect, and they are traditionally utilized couplings (16). Rhubarb is reported to enhance the effect of anhydrous mirabilitum in the intestinal tract and accelerate the onset of purgative action (16). Magnolia bark has psychotropic effects (17), and immature orange has anti-inflammatory effects. Such synergistic effects and multifunctionality are the strong points of Kampo medicine. While the negative effects of polypharmacy may occur to cover various effects with western medicines, the combination of crude drugs in Kampo medicine has been sophisticated throughout history. Considering that many patients in the ICU have systemic inflammatory syndromes and that constipation is associated with delirium in critically ill patients (12), the various effects of DJT may be appropriate in the ICU. 


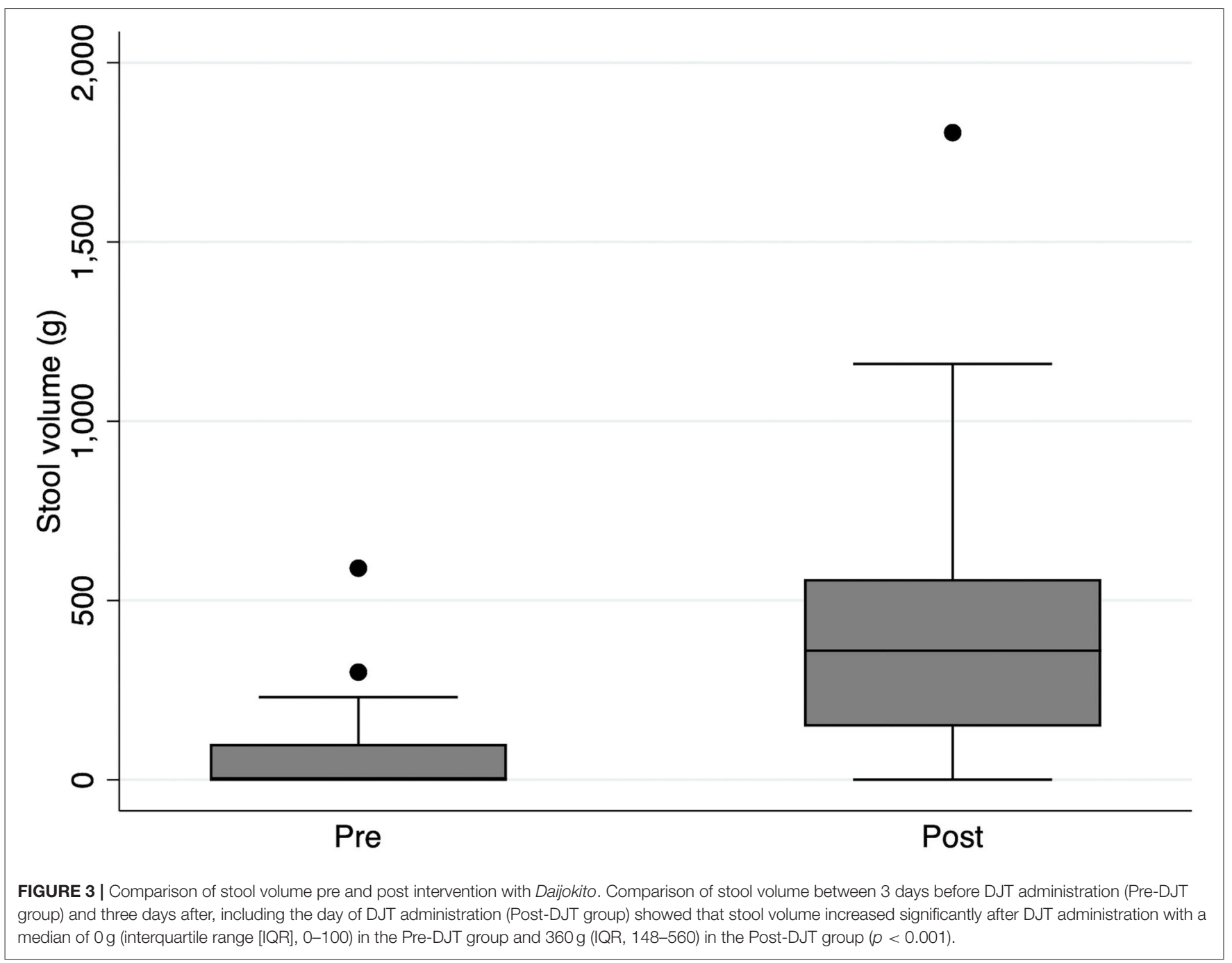

Our study has two limitations. First, the present study was a pre- and post-observational study and did not exclude various biases and confounding factors. And the design of this study did not exclude the possibility that defecation occurred in the natural course without the use of DJT, and that the phenomenon of regression to the mean was observed. Second, the introduction/termination of DJT was left up to the clinician, which was potentially subject to bias. In particular, DJT tends to be initiated in constipation refractory to multiple laxatives, and the major limitation is the inability to distinguish the effects of various laxatives from those of DJT. In addition to the present report, further studies are warranted to quantify the therapeutic efficacy and safety of DJT.

In conclusion, the administration of DJT caused defecation in critically ill patients and significantly increased the stool volume. Although loose unformed stools were the most common stool quality, only one patient had to discontinue DJT administration. Therefore, DJT may improve lower gastrointestinal motility in patients the intensive care unit; however, further high-quality studies are needed to establish the reliability of DJT.

\section{DATA AVAILABILITY STATEMENT}

The raw data supporting the conclusions of this article will be made available by the authors, without undue reservation.

\section{ETHICS STATEMENT}

The studies involving human participants were reviewed and approved by the Ethics Committee of Akita University Hospital. Written informed consent for participation was not required for this study in accordance with the national legislation and the institutional requirements.

\section{AUTHOR CONTRIBUTIONS}

KS designed the study and wrote the initial draft of the manuscript. HN contributed to the conception of the study and critically revised the manuscript. All authors contributed to the article and approved the submitted version. 


\section{REFERENCES}

1. Motoo Y, Seki T, Tsutani K. Traditional Japanese medicine, Kampo: its history and current status. Chin J Integr Med. (2011) 17:85-7. doi: 10.1007/s11655-011-0653-y

2. Zhao J, Tang W, Wang J, Xiang J, Gong H, Chen G. Pharmacokinetic and pharmacodynamic studies of four major phytochemical components of Da-cheng-Qi decoction to treat acute pancreatitis. J Pharmacol Sci. (2013) 122:118-27. doi: 10.1254/jphs.13037FP

3. Nakae H, Kusanagi M, Okuyama M, Igarashi T. Paralytic ileus induced by glyphosate intoxication successfully treated using Kampo medicine. Acute Med Surg. (2015) 2:214-8. doi: 10.1002/ams2.103

4. Nakae $H$, Irie $Y$, Satoh $K$, Kitamura $T$, Kameyama K, Nara T, Okuyama M. Treatment for tetanus applying Kampo medicine: Administration of shakuyakukanzoto. Trad Kampo Med. (2021) 8:130-7. doi: $10.1002 / \mathrm{tkm} 2.1283$

5. Manabe N, Camilleri M, Rao A, Wong BS, Burton D, Busciglio I, et al. Effect of daikenchuto (TU-100) on gastrointestinal and colonic transit in humans. Am J Physiol Gastrointest Liver Physiol. (2010) 298:G9705. doi: 10.1152/ajpgi.00043.2010

6. Itoh T, Yamakawa J, Mai M, Yamaguchi N, Kanda T. The effect of the herbal medicine dai-kenchu-to on post-operative ileus. $J$ Int Med Res. (2002) 30:428-32. doi: 10.1177/1473230002030 00410

7. Committee on Japanese guidelines for nutrition support therapy in the adult and pediatric critically ill patients. Japanese guidelines for nutrition support therapy in the adult and pediatric critically ill patients. J Jpn Soc Intensive Care Med. (2016) 23:185-281. doi: 10.3918/jsicm.23.185

8. Department of Pharmacognosy, Phytochemistry and Narcotics. (DPPN), National Institute of Health Sciences (NIHS) of Japan, national Institutes of Biomedical Innovation, Health and Nutrition (NIBIOHN). STORK. Available online at: http://mpdb.nibiohn.go.jp/stork/ (accessed April 1, 2021).

9. Mostafa SM, Bhandari S, Ritchie G, Gratton N, Wenstone R. Constipation and its implications in the critically ill patient. Br J Anaesth. (2003) 91:8159. doi: 10.1093/bja/aeg275

10. Oczkowski SJW, Duan EH, Groen A, Warren D, Cook DJ. The use of bowel protocols in critically ill adult patients: a systematic review and metaanalysis. Crit Care Med. (2017) 45:e718-26. doi: 10.1097/CCM.00000000000 02315

11. Patanwala AE, Abarca J, Huckleberry Y, Erstad BL. Pharmacologic management of constipation in the critically ill patient. Pharmacotherapy. (2006) 26:896-902. doi: 10.1592/phco.26.7.896
12. Smonig R, Wallenhorst $\mathrm{T}$, Bouju $\mathrm{P}$, Letheulle J, Le Tulzo $\mathrm{Y}$, Tadié $\mathrm{JM}$, et al. Constipation is independently associated with delirium in critically ill ventilated patients. Intensive Care Med. (2016) 42:1267. doi: 10.1007/s00134-015-4050-4

13. Vincent JL, Moreno R, Takala J, Willatts S, De Mendonça A, Bruining H, et al. The SOFA (Sepsis-related Organ Failure Assessment) score to describe organ dysfunction/failure. On behalf of the working group on sepsis-related problems of the European society of intensive care medicine intensive. Care Med. (1996) 22:707-10. doi: 10.1007/BF01709751

14. van der Spoel JI, Oudemans-van Straaten HM, Kuiper MA, van Roon EN, Zandstra DF, van der Voort PHJ. Laxation of critically ill patients with lactulose or polyethylene glycol: A two-center randomized, double-blind, placebo-controlled trial. Crit Care Med. (2007) 35:2726-31. doi: 10.1097/01.CCM.0000287526.08794.29

15. Luft VC, Beghetto MG, de Mello ED, Polanczyk CA. Role of enteral nutrition in the incidence of diarrhea among hospitalized adult patients. Nutrition. (2008) 24:528-35. doi: 10.1016/j.nut.2008. 02.004

16. Doui M, Ando H, Goi C, Kakiuchi N, Mikage M. Herbological studies on combination of rhubarb and mirabilite. Yakushigaku zasshi. (2010) 45:106-11.

17. Koike H, Yoshino T, Nakazawa A, Horiba Y, Adachi T, Watanabe K. Two elderly cases of constipation with irritability diagnosed as alzheimer's dementia after successfully treated with daijokito. Kampo Medicine. (2019) 70:219-26. doi: 10.3937/kampomed.70.219

Conflict of Interest: The authors declare that the research was conducted in the absence of any commercial or financial relationships that could be construed as a potential conflict of interest.

Publisher's Note: All claims expressed in this article are solely those of the authors and do not necessarily represent those of their affiliated organizations, or those of the publisher, the editors and the reviewers. Any product that may be evaluated in this article, or claim that may be made by its manufacturer, is not guaranteed or endorsed by the publisher.

Copyright (c) 2021 Satoh and Nakae. This is an open-access article distributed under the terms of the Creative Commons Attribution License (CC BY). The use, distribution or reproduction in other forums is permitted, provided the original author(s) and the copyright owner(s) are credited and that the original publication in this journal is cited, in accordance with accepted academic practice. No use, distribution or reproduction is permitted which does not comply with these terms. 